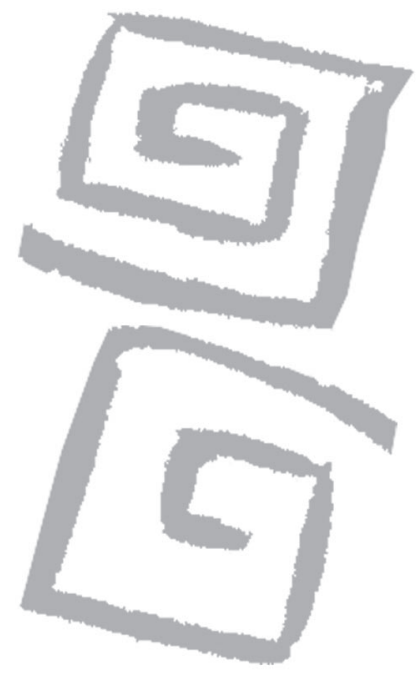

\title{
Las estadísticas de salud no nacen de un repollo: jesuitas, aritméticas políticas, estigmergias y oligópticos
}

\author{
Health statistics aren't born in a cabbage patch: \\ Jesuits, political arithmetic, stigmergy and oligopticons
}

'Médico-Cirujano, Doctor en Salud Pública. Profesor asistente, Escuela de Salud Pública, Universidad de Chile. Santiago, Chile. ycarvajal@med.uchile.cl

${ }^{2}$ Licenciado en Filosofía, Doctor en Filosofía. Profesor, Universidad Mayor, Chile. tuillang@yahoo.com
Carvajal, Yuri'; Yuing, Tuillang ${ }^{2}$

RESUMEN A partir de un análisis del contenido y la red productora de un mapa de 1751, elaborado por la misión circular de los jesuitas en Chiloé (archipiélago ubicado en la costa austral chilena), que contiene cifras de nacimientos, defunciones y habitantes, este artículo discute el rol de las estadísticas de salud, en diferentes registros: históricos, filosóficos, técnicos y sociológicos. De este modo, se busca comprender la génesis de un proceso de producción de cifras y referencias, para debatir de qué están hechas las estadísticas de salud, qué roles cumplen, sus vínculos con la construcción de colectivos y las condiciones de posibilidad de producciones diferentes. Tratamos de ensayar algunas hipótesis que muestran las estadísticas como articulación híbrida de elementos de índole muy diversa: epistemológicos, biopolíticos, históricos y filosóficos, reconociendo en ellos facetas religiosas y demográficas, ontológicas y étnicas, científicas y de gobierno.

PALABRAS CLAVES Estadísticas Vitales; Cartografía; Chile.

ABSTRACT By analyzing the content and network of production of a map from 1751, created by the circular mission of the Jesuits in Chiloé (an archipelago located off the southern coast of Chile), that contains birth, death and population data, this article discusses the role that health statistics play historically, philosophically, technically and sociologically. In doing so, the article seeks to comprehend the genesis of a process of production of data and references in order to debate what health statistics are composed of, what ends they are used for, what their connection is to the formation of collectives and the differential conditions of possibility that exist for producing statistics. We attempt to develop hypotheses that demonstrate statistics as a hybrid articulation between diverse elements, epistemological, biopolitical, historical and philosophical in nature, with facets at once religious and demographic, ontological and ethnic, scientific and governmental.

KEY WORDS Vital Statistics; Cartography; Chile. 


\section{ESTADÍSTICAS COMO PREGUNTAS}

La discusión y el debate sobre los modos de ser y existencia de las estadísticas no se restringen a una lógica unívoca. Entre muchos protocolos de aproximación, es posible llevar a cabo un análisis con énfasis teórico que atienda a los supuestos y alcances ontológicos y epistémicos de las cifras y sus sintaxis. Pero también es posible llevar a cabo esta reflexión a partir de un objeto material -empíricamente acotado- cuyas distancias de uso permiten una exploración de la materialidad y sus implicancias en la configuración de las estadísticas como objeto tecnocientífico.

En nuestro caso, proponemos al lector un recorrido que se inclina por el segundo sentido, a partir del estudio de un mapa que tiene 260 años a sus espaldas. La confección de este mapa incluye cifras de lo que hoy llamamos estadísticas vitales, que están referidas al archipiélago de Chiloé en la época colonial chilena. Se trata, además, de un territorio distante de los centros de gobierno no solo en un sentido temporal y geográfico. A ello se suma un modo de vida navegante y nómada posibilitado por un mar interior que resquebraja y desordena tanto el territorio como la administración. La conjunción de estos elementos constituye el punto de partida para esta investigación.

\section{EL MAPA DE 1751}

Al examinar la Figura 1, la atención es capturada por el mapa del centro. El trazado perfila un contorno alargado y curvo de la isla de Chiloé, diferente de la representación actual. Un número correlativo identifica 76 lugares que hoy igualmente son localidades habitadas. Una línea discontinua describe un trayecto que enlaza los 76 sitios. A la izquierda del mapa -una sola gran columna- se encuentra una enumeración en latín con 15 párrafos. A la derecha, se advierte una tabla de 77 filas y 7 columnas. Los encabezados de la tabla se enuncian con palabras que nos suenan conocidas: oratoria, familias, animas, comuniones, baptismos, matrimonios, defunciones. La última línea: summa; y, por supuesto, al pie de la tabla, explicaciones y descripciones de las categorías usadas. Cada columna representa lugar, familias, personas, matrimonios, nacimientos y defunciones de los sitios representados en el mapa y al pie, los totales. Finalmente un texto.

Mirando simultáneamente el mapa y las tablas, nos enteramos, por ejemplo, que el primer lugar marcado es Ichuac, con 75 familias, 370 animas, 426 comuniones, 24 baptismos, 2 matrimonios y 12 defunciones. El último lugar es Castro. En total: 2.295 familias, 11.647 animas, 12.720 comuniones, 626 baptismos, 113 matrimonios y 515 defunciones.

Se trata no solo de un excelente registro de estadísticas vitales -con todas las variables que actualmente definen este campo- sino además georeferenciado. Todo un paper, si no fuera por el formato en un pliego, la falta de referencias en formato Vancouver o APA; carece de resumen y por supuesto, de palabras claves. ¿La filiación de los autores? Son los jesuitas de la Residencia de Chiloé, en Castro (centro de la isla) que informan de la misión circular realizada en 1751 (Figura 2).

¿Cómo ha sido posible esta producción científica de estadísticas vitales en la oscura colonia chilena y tan lejos de la sede monárquica?

\section{LA ACTIVIDAD DE LOS JESUITAS}

\section{Misiones circulares}

El mapa informa de una de las tantas misiones circulares que los jesuitas emprendían todos los años en el mes de septiembre, hasta mayo del año siguiente, desde 1624 hasta su dolorosa expulsión el 8 de diciembre de 1767 (1). Navegando en dalcas chilotas (a), los padres tenían como misión principal convertir al cristianismo, pero también destinaban tiempo a la construcción de iglesias, recolección y registro de cantos, conocimiento herbolario, y la exploración de pasos como, por ejemplo, el de los Vuriloches que conecta el Estuario de Reloncaví con la Patagonia argentina. Las cifras registradas tenían connotaciones proselitistas y de protección de los indios. Pero a la vez expresaban el empeño de una tarea performativa que vale la pena subrayar y destacar: mediante el uso de tablas, números y gráficos, hacer de estos chonos (b) errantes un colectivo. Se trata de la puesta en forma de un territorio codificado, organizado, diagramado: 


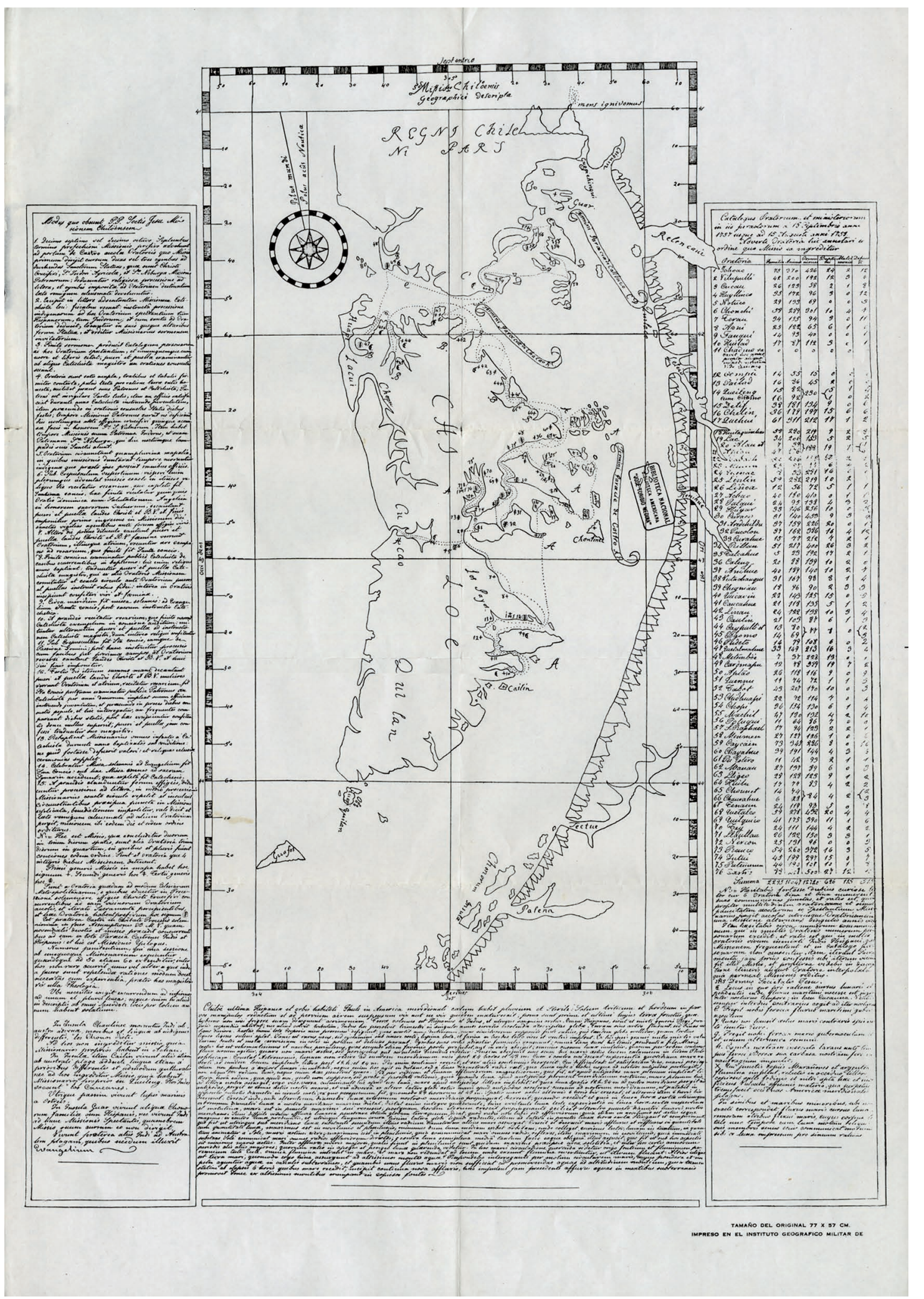

Figura 1. Misión Circular de los jesuitas de Chiloé del año 1751. 
Refidencia de Chiloe.

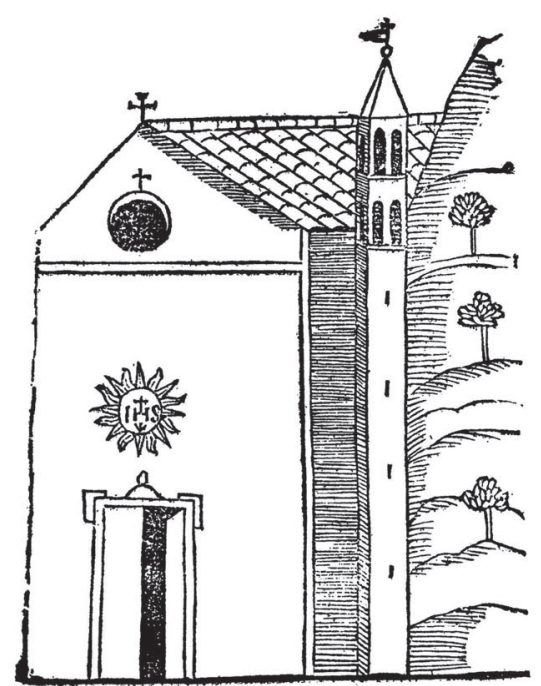

TE Sez Refidencia es la corona de todas las miffiones, de donde falen nueftros $\mathrm{Pa}$ piraguas, con tan grandes yocomodidades, trabajos, y pelioros dela vidata, que no ay on ifrion, que en efto is exceda, falen tambien a tierry firme, de dunde tiene que correr de largo mas de cien leguas, hafta eleftrechode Magallianes.

Figura 2. Imagen de la residencia de los jesuitas en Castro según Alonso de Ovalle, 1646.

Fuente: Alonso de Ovalle (2).

Nota: El año indicado corresponde a la primera edición del libro de Alonso de Ovalle, publicado en Roma.

La misión más trabajosa que aquí tienen los padres es la de los chonos, gente más apartada del comercio de los españoles, más cercana al Estrecho e inculta de cuantas hay en estas partes. Divídense en varias parcialidades esparcidas por muchas islas, como en el archipiélago de Chiloé. No tienen morada cierta, de continuo traen el hato a cuestas, mudándose con su familia de isla en isla a coger marisco, que es su ordinario sustento, sin tener otras chácaras ni sementeras; a que añaden beber el aceite de lobos, con que traen el color pálido, y a la causa viven lo más del año dentro del mar, porque les es fuerza buscar en él su sustento. ( 2 p.555) [cursivas del original]

¿Cómo comprender el esfuerzo múltiple de los jesuitas? ¿Qué aspectos se dan cita en este ejercicio diverso de evangelización y civilización? ¿Cómo rescatar los distintos enfoques que intervienen en esta conformación primaria de territorialización, gobierno y población? ¿Cuáles son sus supuestos científicos y disciplinarios?

Mapa, territorio, gubernamentalidad

Vale la pena entonces un recorrido en torno a los puntos de encuentro entre mapa y territorio. En efecto, la proposición el mapa no es el territorio, "hecho célebre por Alfred Korzybski" (3 p.26), alude a que la cartografía y elaboración gráfica dista del paisaje. Basta asomarse por aquellas fronteras nacionales situadas en terrenos hostiles como la cordillera o el desierto, para dar cuenta de la imposibilidad de encontrar, en el bello paisaje salvaje, algo así como un señalamiento, una división u otro dispositivo definitorio de la soberanía. La sentencia invita a no confiar plenamente en la representación de lo que se explora y evitar de ese modo el riesgo del extravío. Pero no resta al mapa su sentido ordenador, su pretensión de control: en busca de la orientación, el mapa es un intento de dominio, de gestión, de organización. Por ello, más que perseguir la inmediata cercanía de un paisaje virgen y salvaje, la proposición acusa la consideración de que todo mapa es un ensayo de la voluntad -deliberado o no-, un intento de conformar, inventar y fundar un territorio. En ese sentido, el mapa es, entonces, territorialización: una codificación que permite el avance, el ordenamiento, la delimitación de algo que asoma como desconocido.

Deleuze y Guattari (4) han entregado algunos alcances en la conceptualización de la territorialización, entendida como un permanente movimiento de despliegue y repliegue de la subjetividad en la diversidad de sus prácticas. Con este lenguaje, estos autores buscan entregar herramientas de explicación de los procesos políticos y culturales sin apelar a la sustancialidad del sujeto ni a procesos estructurales invariantes que proceden con lógicas binarias, en beneficio de una lógica rizomática y de flujos, que enfatiza la movilidad y pluralidad de las dimensiones que intervienen en estos procesos. Así se apela a una complementariedad más que a una oposición entre modelos explicativos, en las que, sin embargo, el principio territorial es ineludible: 


\begin{abstract}
...uno actúa como modelo y como calco trascendente, incluso si engendra sus propias fugas; el otro actúa como proceso inmanente que destruye el modelo y esboza un mapa, incluso si constituye sus propias jerarquías... (4 p.25)
\end{abstract}

Desde esta perspectiva, el mapa interroga y trata de establecer un punto de contacto con las propias referencias, con la propia simbología que permite entender el mundo y ordenar la experiencia. Pero no se trata únicamente de un gesto interpretativo. La geografía está siempre asociada a un impulso conquistador, y así la territorialización señala el despliegue de una subjetividad en avance:

\footnotetext{
...el territorio es sinónimo de apropiación, de subjetivación fichada sobre sí misma. Él es un conjunto de representaciones las cuales van a desembocar, pragmáticamente, en una serie de comportamientos, inversiones, en tiempos y espacios sociales, culturales, estéticos, cognitivos. (5 p.467-468)
}

En definitiva, pese al carácter pasivo de las misiones jesuitas, el mapa es indicativo del encuentro entre las pretensiones jesuitas de conversión religiosa del pueblo chono, de existencia nómade en una zona de mar interior. Es el intento de articular un mundo común entre habitantes de mundos disímiles. Por otra parte, Foucault ha descrito esta dinámica en términos de gubernamentalidad. En efecto, el proceso mediante el cual la lógica territorial da paso a una lógica de la población, permite comprender la importancia de la gestión y el ordenamiento del territorio para la conformación de una población:

Entiendo por gubernamentalidad, el conjunto constituido por instituciones, procedimientos, análisis y reflexiones, cálculos y tácticas que permiten ejercer esta forma, tan específica como compleja, de poder, que tiene como objetivo principal la población, por forma mayor de poder, la economía política, por instrumento técnico esencial, los dispositivos de seguridad. (6 p.655) [traducción nuestra] (c)

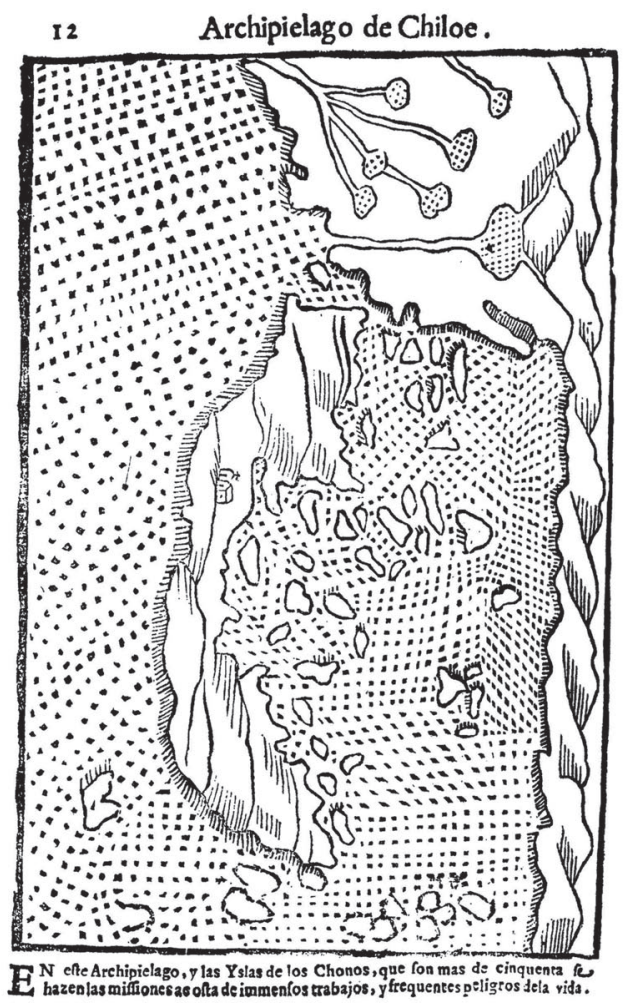

Figura 3. Mapa de Chiloé según Alonso de Ovalle, 1646.

Fuente: Alonso de Ovalle (2)

Nota: El año indicado corresponde a la primera edición del libro de Alonso de Ovalle, publicado en Roma.

Como buena parte de las nociones que Foucault utiliza, las de territorio y población, más que anularse o desplazarse, se suman, complementan y superponen, cambiando acentos y aguzando los enfoques. Así, la gubernamentalidad supone el gesto previo del ordenamiento y gestión territorial en términos de límite y soberanía, pero va de la mano con la elaboración estadística y el inventario de los elementos que se disponen en el espacio. Se trata, más precisamente, de un arte de gobernar que elabora una racionalidad y unos procedimientos en vistas al funcionamiento del Estado. Más que desconocer el territorio, se trata de su administración, del conocimiento de sus fuerzas y sus particularidades en orden a un proyecto, a un ejercicio de gobernabilidad. Este mapa contempla variables de gobierno, índices de población y una aproximación tentativa al estado de estos indios en la 
fe. En suma, una primaria puesta en limpio de sus modos de vida. El valor de los mapas para los jesuitas explica que existan varias representaciones de estas misiones y también, que los documentos que registran la labor confesional de la orden, sean relaciones históricas con una gran producción cartográfica, como muestra la Figura 3, tomada del libro de Ovalle.

\section{Aritméticas políticas}

El uso de cifras para producir colectivos puede tener alguna relación con lo que Foucault Ilamaba técnicas de gubernamentalidad $(7,8)$. La expresión quizá enfatiza excesivamente el aspecto de gobierno desde una perspectiva vertical y relega problemas más bien horizontales, de referencia, de traducción y de coordinación necesarios en todo colectivo, a un examen en la perspectiva de instituciones más o menos centralizadas que se imponen, en vez de reconocer formas más bien distribuidas y de doble flujo. En efecto, Foucault aborda la gubernamentalidad desde una perspectiva adjetivada como biopolítica, como una tecnología que acusa una específica forma de ejercicio del poder sobre la vida de los individuos y que marca, fundamentalmente, el despliegue de las políticas de Estado sobre la población. Desde luego, así señalada y puesta en vecindad con las tecnologías disciplinarias, la gubernamentalidad no goza de buen semblante, más bien adquiere sospechosas connotaciones de dominación y control que, si bien tiene sentido, no debe hacer olvidar que la implementación y elaboración de la población como objeto de gobierno representa el momento que posibilita la visibilización de carencias, la preocupación por las condiciones de vida y, en ese sentido, la conformación de las garantías sociales básicas. Se trata de una discusión que, a nuestro juicio, descuida la riqueza performativa del gesto técnico contenido en el mapa, y no permite vislumbrar el valor que específicamente guarda para las estadísticas de salud. No hay aquí un juicio de la ética o "militancia" de la racionalidad e intencionalidad susceptible de revelar este trazado jesuita.

Ahora bien, quizá los salubristas tenemos en mente, cuando hablamos de números y gobierno, a William Petty. Desde luego pensamos en el autor de los Natural and Political Observations; mentioned in a following Index, and made upon the bills of mortality, trabajo presentado bajo la firma de John Graunt en la Royal Society de Londres en 1662, en el que la modelación matemática de la dinámica poblacional, a partir de los registros parroquiales de Londres, le permite en un mismo movimiento calcular los habitantes de la ciudad y tener como interlocutor al Rey.

Pero Petty puede ser pensado además como precursor del movimiento fisiocrático. Un renovador del mercantilismo que, enfatizando la agricultura y la productividad de los campesinos, propone una transformación intelectual de la concepción de lo económico -liderada por Turgot y Quesnay- alrededor del problema del valortrabajo (9). Aspectos claves que hasta hoy animan el pensamiento económico y, como tal, son articuladores de la economización de la vida colectiva. Las palabras con que su hijo introduce la edición póstuma de Political Arithmetick de Petty en 1690 destacan su propósito:

Fue por él denominada Aritmética Política, ya que las materias de gobierno, la Gloria del Príncipe, la felicidad y grandeza del pueblo, son por las reglas comunes de la aritmética, transformadas en una suerte de demostración. (10 p.3) [traducción nuestra] (d)

Los números para Petty son instrumentos de gobierno pero también de felicidad del pueblo, de debate y demostración. Los números como construcción del colectivo en la vida de Petty estuvieron presentes en el censo de Irlanda, en las cifras de mortalidad y en el desafío de construir cuentas nacionales. Con todo, lo que Petty hizo en el siglo XVII, nuestros jesuitas lo hicieron un siglo después, cimentando las bases de lo que vivimos como un fenómeno de economización de la vida colectiva, bajo reglas de lo que Callon Ilama una antropología neoclásica: "to higlight the fact that any economics is an antropologics" (11 p.10), pues supone un cierto modelo de lo humano, el de un alguien que es un sujeto, que está individualizado y, a la vez, es portador de una racionalidad económicamente organizada.

El movimiento de las aritméticas políticas y estadísticas tenía en ese momento algunas décadas de historia: 
Se considera generalmente al profesor alemán Hermann Conring (1606-1681) como el primero en impartir cátedra de este género. Gottfried Achenwall (1719-1772), otro profesor alemán de la misma tendencia, introdujo el término "estadística". Las "estadísticas" que estos profesores manejaban estaban principalmente consagradas a la presentación de datos no numéricos y no tenían, pues, nada en común con lo que hora llamamos método estadístico. No obstante, el objetivo que pretendían era en buena parte semejante al de nuestros cuadros numéricos, aunque éstos estén indudablemente elaborados con métodos un tanto más refinados. (12 p. 201)

Una reflexión sobre ambos aspectos de las estadísticas, gubernamentalidad y economización, es de urgente actualidad para la salud pública. El creciente proceso de economización de la salud y la necesidad de abordar directamente los aspectos políticos de la salud colectiva requieren examinar objetos tan próximos y de tanto poder como las estadísticas, con un primer gesto de extrañeza (e).

Cuando decimos economización de la vida colectiva, apuntamos al uso de dispositivos de cálculo que articulan dominios de la vida como fenómenos económicos (18). El estudio de la economización es un área de trabajo de la sociología de la traducción o actor network theory que busca comprender cómo se entrelazan (entanglements y disentanglements) objetos y personas en estos agenciamientos y buscando así condiciones de posibilidad para otras variantes de numeración del colectivo (11).

\section{CIENCIA MODERNA}

La forma en que los números trabajan en el surgimiento de la ciencia contemporánea tiene estrechas vinculaciones con el significado divino de las cifras. Como demuestra Frances Yates (19), la herejía de Giordano Bruno (1548-1600) no solo era desbancar una teoría astronómica equivocada. Se vinculaba con el diálogo pagano del cristianismo del siglo II del Asclepio y el Pimander (f), equivocadamente atribuidos a los tiempos precristianos. Ubicar el sol en el centro del universo era un giro que además ubicaba a los números y a los signos en una perspectiva distinta. El uso de la mnemotecnia y su trabajo con esquemas espaciales para tales propósitos también es parte de su legado intelectual. Bruno habla de mathesis divina, una de las cuatro guías de la religión (19). Esta mathesis encuentra su eco en el proyecto de la mathesis universal, que según Foucault, se desarrolla durante el siglo XVII y XVIII (20).

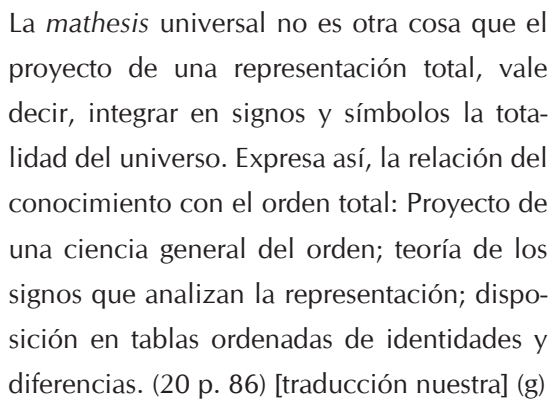
proyecto de una representación total, vale decir, integrar en signos y símbolos la totalidad del universo. Expresa así, la relación del conocimiento con el orden total: Proyecto de una ciencia general del orden; teoría de los signos que analizan la representación; disposición en tablas ordenadas de identidades y diferencias. (20 p. 86) [traducción nuestra] (g)

En todo caso, es una noción que engloba muchas acepciones y que no se encuentra de manera totalmente explícita. Antes bien, la mathesis circula como aspiración que se manifiesta en un método de análisis universal, que integra dentro de su esquemática todo tipo de naturalezas simples, vale decir, todo caso particular. La aproximación a cualquier tipo de singularidad, ya sea en el ámbito de los seres vivos, en el de las palabras y nombres o en la estimación del valor monetario -en definitiva todo caso- se encuentra mediada por un diseño general del orden en el que tiene lugar y gracias al cual además se representa. Por la misma razón, la mathesis siempre mantiene una relación estrecha con una génesis -determinación del origen y posibilidad de la comparación- y con una taxinomia -analítica de las representaciones con base en sus similitudes y diferencias- que permiten finalmente la elaboración de un sistema de signos en el que es posible leer el continuum de las cosas. Luego, se asiste a un método de lectura del gran orden de la naturaleza, una clave de análisis que establece una disposición certera entre las representaciones y los signos. Una clave, por cierto, que se enarbola como marco en el que se dan cita la gramática general, la historia natural y el análisis de las riquezas y que, en su conjunto, se constituye como un reflejo del orden del mundo: 
El continuo de la representación y el ser, una ontología definida negativamente como ausencia de nada, una representabilidad general del ser, y el ser manifestado por la presencia de la representación -todo esto es parte de la configuración de conjunto de la episteme clásica. (20 p.219) [traducción nuestra] (h)

La referencia a los números, un lugar central en la ciencia actual, fue a su vez potenciada y derivada a nuevas implicancias por el uso de la imprenta de tipos móviles y la posibilidad de contar con miles de copias, réplicas similares de un mismo texto (21). Goody señala que, en colectivos donde la difusión de la escritura es restringida, la magia impregna la interpretación de los números (22). Podríamos considerar que a partir de la imprenta, la circulación de textos escritos tampoco se masificó lo suficiente para evitar la magia de los números.

Ahora bien, es precisamente esa magia la que continúa presente en el mundo jesuita. En efecto, podemos encontrar influencia de Bruno sobre Athanasius Kircher (sacerdote jesuita alemán), quien en sus obras menciona a Bruno y como él, cree aún en la filiación egipcia del cristianismo de acuerdo con la atribución errada del Asclepio y el Corpus Hermético.

Como ha demostrado Acuña (23), el sacerdote jesuita chileno Alonso de Ovalle, en su visita al Vaticano entre 1647 y 1650, tuvo un importante contacto con Kircher, quien publicó materiales de Ovalle en dos de sus libros. La presencia e importancia de Kircher entre los jesuitas chilenos se expresa en 18 ejemplares de sus libros que pasaron a la Universidad Real de San Felipe (primera universidad de Chile) y el 5 de agosto de 1818 a la Biblioteca Nacional de Chile. Nicolás Mascardi, discípulo predilecto de Kircher, se une a Ovalle en su retorno a Chile y nunca volvería a Italia. Existen siete cartas enviadas por Mascardi a Kircher que dan cuenta de la mantención del vínculo pese a la distancia.

Mascardi tenía conocimiento de matemáticas y un particular interés por la geografía y, junto con Alonso de Ovalle y otro joven estudiante, José María Adami, visitaron a Giovanni Battista Riccioli, jesuita italiano estudioso de la geografía. Tras la muerte de Mascardi entre sus pertenencias se hallaba la Astronomia de Riccioli.
Mascardi llegó a Chile en 1652 y tomó el camino de misionero en la villa de Buena Esperanza en la Región de Araucanía. Destruidos sus instrumentos en la insurrección acaudillada por Tinagucupu en 1655, es nombrado rector del colegio jesuita en Chiloé en 1662. Sabemos que este discípulo de Kircher muere en la búsqueda de la Ciudad de los Césares en 1674, en un viaje desde Castro hacia el este, cruzando la cordillera por el Estuario de Reloncaví a través del paso de los Vuriloches.

El conocimiento técnico y la capacidad de dibujar y contar de los jesuitas de Chiloé, tiene pues un punto común con el surgimiento de las ciencias modernas y el rol de los números. De alguna manera, todos estos elementos: la mathesis, la concepción mágica de los números, la influencia de Giordano Bruno, están presentes en el trabajo y diseño territorial que los jesuitas hicieron en Chiloé. Por otra parte, esto relativiza los juicios acerca de qué es centro y qué periferia, en un momento dado. Estamos tan acostumbrados a juzgar de acuerdo a la narración de los triunfadores (historia whiggish), como si el territorio nunca hubiera estado en disputa por líneas de fuerzas diversas, como si existiera un centro establecido.

\section{ESTADÍSTICAS Y ESTIGMERGIA}

Las cifras del mapa nos permiten discutir algunas de las explicaciones sobre el surgimiento de las estadísticas. Hervé Le Brass ha cuestionado las formulaciones que analizan el uso de las cifras como forma de organizar colectivos apelando a una mera explicación económica o una racionalidad capitalista (9). Le Brass no acepta que las tablas de mortalidad se relacionen con un cálculo supuestamente racional para los seguros, ni que tengan su origen en las tablas de doble entrada de la contabilidad. La crítica de Le Brass es importante porque entronca con radicales cuestionamientos acerca de la utilidad real de usar la palabra "capitalismo", como el de Goody:

\footnotetext{
...iporqué no prescindimos de ese término peyorativo que data de la Inglaterra del siglo XIX y reconocemos el elemento de continuidad
} 
en el mercado y en las actividades de la burguesía desde la edad de bronce hasta épocas recientes? (24 p.227)

O como el de Latour:

...el capitalismo no tiene enemigo posible puesto que está en "todas partes" pero una determinada sala de operaciones bursátiles en Wall Street tiene muchos competidores en Shangai, Frankfurt y Londres -la rotura de una computadora, un movimiento traicionero de un competidor, una cifra inesperada, una variable desatendida en una fórmula de fijación de precios, un procedimiento contable riesgoso- que puede hacer pasar el balance de una ganancia obscena a una pérdida dramática [...] no nos centremos en el capitalismo, pero tampoco nos quedemos fijados a la pantalla de la sala de operaciones bursátiles: sigamos a las conexiones, "sigamos a los actores mismos". (25 p.256-257) [cursivas del original]

A partir de algunas de las investigaciones de Foucault, Senra propone comprender las estadísticas como tecnologías de distancia (26) y Desroiséres como una combinación de gobierno y medida (27). Senra reconoce junto con las funciones semánticas o representacionales usuales de las cifras, una función sintáctica de las estadísticas, es decir, organizadora y performativa. Este bifrontismo de los números, que permite examinarlos con miradas más ricas, adolece empero de un estilo de análisis basado en la sospecha y que nos devuelve a la cuestión de la gubernamentalidad.

Que había cuestiones de gubernamentalidad en el mapa de los jesuitas y en las acciones de la orden, es innegable. Estimaciones contemporáneas señalan que los jesuitas controlaban algo así como el $25 \%$ del producto bruto interno de lo que era Chile en ese entonces, constituyendo una especie de gobierno en el inxilio -interno, pero a la vez de una alteridad con un antagonismo pasivo- que por supuesto inquietaba al Rey, toda vez que conocía las fuerzas políticas renovadoras en América y en Europa (28).

Pero hay algo más que gubernamentalidad en las cifras. Hay también aspectos colectivos incuestionables, lo que hemos llamado la dimensión horizontal de los números, que permiten hacer vida en común. Latour ha propuesto tomar la expresión estigmergia usada en ecología $(29,30)$, haciéndola extensiva a los humanos.

Surgida de los estudios de los animales que se organizan en colectivos, la estigmergia se refiere a las señales trazadas sobre las paredes de las colmenas, sobre los nidos o en los acúmulos de materias, que guían el trabajo de los animales que continúan la obra. Esas señales permiten coordinar la producción común. Para aquel que no considera la estigmergia, el todo parece emerger como algo inexplicablemente surgido de un caótico agregado de partes. Un colectivo que surge de la acción espontánea de individuos aislados. Pero la estigmergia hace innecesaria la invisible mano de Dios en las colmenas.

Los holismos y los atomismos en sociología contraponen individuo a sociedad. Pero esa agonía desaparece si consideramos que no hay dos niveles en juego, el individuo y el todo, sino un continuo articulado por trazas estigmérgicas.

En el mundo humano, enriquecido y multiplicado por esos objetos técnicos que son el lenguaje oral, la escritura, los números, el cero, los gráficos, la imprenta, los computadores e Internet, los objetos estadísticos cumplen un rol que nos atrevemos a Ilamar estigmérgico. El mapa propone articular formas organizativas de la vida de un colectivo, señalar importancias, tener problemas en común, realizar debates. Son formas por supuesto contingentes y transitorias, sin carácter de necesidad ni organizadas en torno a una ruptura radical con ningún pasado lineal.

\section{OLIGÓPTICOS}

La producción de estadísticas en salud es parte de esta función estigmérgica, que nos conecta con las expresiones de Dewey acerca de la democracia entendida como la búsqueda de la construcción de un público y el rol que en esa tarea correspondía a los signos y al arte:

Nuestra babel no es de lenguas, sino de unos signos y símbolos sin los cuales es imposible 
la experiencia compartida [...] Sólo cuando existen signos o símbolos de las actividades y de su resultado, el flujo puede contemplarse desde el exterior, puede aislarse para considerarlo y juzgarlo, y, en consecuencia, puede llegar a regularse. Los rayos pueden golpear y partir un árbol o una roca, y los fragmentos resultantes retomar y continuar el proceso de interacción. Pero cuando las fases del proceso son representadas a través de unos signos, se interpone un nuevo medio. Como los símbolos se relacionan mutuamente, las relaciones importantes de un curso de acontecimientos se registran y se conservan como significados. La memoria y la previsión se hacen posibles; el nuevo medio facilita el cálculo, la planificación y el nuevo tipo de acción que interviene en lo que sucede para dirigir su curso en interés de lo que se prevé y se desea. $(31$ p.134,141)

Como buen discípulo de Peirce, Dewey comprendía las funciones indispensables del signo, en su variante palabra, número, ícono, en la vida colectiva. Su valoración del signo en la constitución de un público es concordante con la visión que del mismo tenía Peirce:

Es que la palabra o signo que utiliza el hombre es el hombre mismo. Pues lo que prueba que un hombre es un signo es el hecho de que todo pensamiento es un signo, en conjunción con el hecho de que la vida es un flujo de pensamiento [...] El hombre individual, dado que su existencia separada se manifiesta sólo por la ignorancia y el error, en la medida en que es algo aparte de su prójimo, y de lo que van a ser él y ellos, es sólo negación. (32 p.121)

Un lugar que donde se reúnen las inscripciones (o signos) y se preserva su trazabilidad, es decir su cadena de transformaciones, puede ser considerado un oligóptico:

\begin{abstract}
...esos sitios que hacen exactamente lo opuesto de los panópticos: ven demasiado poco como para alimentar la megalomanía del inspector o la paranoia de los inspeccionados, pero lo que ven lo ven bien; de allí el uso de esta palabra griega para designar un ingrediente al mismo tiempo indispensable y que viene en cantidades minúsculas [...] Desde los oligópticos es posible obtener visiones sólidas pero extremadamente restringidas del todo (conectado), mientras se mantengan las conexiones. (25 p.260) [cursivas del original]
\end{abstract}

Los jesuitas en su mapa trazaron un oligóptico chilote, juntaron en una sola gráfica bivariada sobre una superficie de papel, lo que a ellos les parecía "importante"' de los chonos. La cuestión de la importancia en estadísticas es de primera relevancia. Como decía Whitehead:

Concentramos nuestra atención gracias al sentido de la importancia. Y al hacerlo, atendemos a los hechos. Aquellos que de un modo deliberado limitan su atención a los hechos lo hacen por razón del sentido de la importancia que otorgan a aquella actitud. Ambas nociones son antitéticas y se requieren mutuamente. (33 p.14)

Este documento releva hoy otros sentidos que en ese momento no fueron considerados, como por ejemplo el valor patrimonial arquitectónico de las construcciones y lugares enumerados en el mapa. Si bien es cierto que el oligóptico ve menos que lo que hay, lo cierto es que permite ver simultáneamente lo que de otro modo no veríamos. Algunos Ilaman a esos oligópticos "el todo", el diagnóstico de salud, la situación de salud, las prioridades de salud, las estadísticas de salud. No hay problema, no estamos discutiendo nombres. Solo que insistimos que ese todo es siempre más pequeño que las partes y que ese todo es contingente y uno más, entre muchos todos posibles. Otras estadísticas no solo son necesarias. Queremos pensar que son cada vez más posibles. 


\section{NOTAS FINALES}

a. Las dalcas son embarcaciones de tres tablas cocidas y calafateadas con estopa de alerce, dan cuenta de la hibridación que constituyen las misiones circulares.

b. Los chonos constituían un pueblo navegante en ese territorio insular y conformaron una especie de cultura maderera, cuyo producto más representativo fueron las canoas de tres tablas -dalcas-, y las actuales iglesias chilotas patrimonio de la humanidad. Las Misiones Circulares jesuitas en Chiloé articularon el encuentro religioso, político y material con esta etnia.

c. "Par gouvernementalité, j'entends l'ensemble constitué par les institutions, les procédures, analyses et réflexions, les calculs et les tactiques qui permettent d'exercer cette forme bien spécifique, bien que complexe, de pouvoir, qui a pour cible principale la population, pour forme majeure de savoir, l'économie politique, pour instrument technique essentiel les dispositifs de sécurité" (8 p.655).

d. "It was by him stiled Political Arithmetick, in as much as things of Government, and of no less concern and extent, than the Glory of the Prince, and the happiness and greatness of the People, are by the Ordinary Rules of Arithmetick, brought into a sort of Demonstration" (10 p.3).

e. Con otras palabras y otros enfoques, Adolfo Murillo (13), Augusto Orrego Luco (14), Salvador Commentz (15), Alvaro Covarrubias (16) y Hugo Behm (17) discutieron sobre estadísticas con un afán colectivo.

f. Pimander es el primer tratado del Corpus Hermeticum, que junto con el Asclepio son textos producidos entre los años 100 a 300 d.c., muy debatidos en el Renacimiento, bajo la convicción de que procedían de la civilización egipcia.

g. "Projet d'une science générale de l'ordre; théorie des signes analysant la représentation; disposition en tableaux ordonnés des identités et des différences..." (20 p. 86).

h. "Le continuum de la représentation et de l'être, une ontologie définie négativement comme absence de néant, une représentabilité générale de l'être, et l'être manifesté par la présence de la représentation, -tout ceci fait partie de la configuration d'ensemble de l'épistémè classique." (20 p.219).

\section{AGRADECIMIENTOS}

Agradecemos a Ana María Adriazola y a sus colegas del Museo Nacional de Medicina Universidad de Chile, por la digitalización de la imagen. Yuri Carvajal reconoce el financiamiento del Conicyt (Fondecyt 3130585) a través del proyecto "Controversias tecno-científicas en la reforma de salud: análisis desde la sociología de la traducción".

\section{REFERENCIAS BIBLIOGRÁFICAS}

1. Tampe Maldonado E. Tres siglos de misiones en Chiloé. Santiago de Chile: Editorial Salesiana; 1981.

2. Ovalle A. Histórica relación del reino de Chile. Santiago de Chile: Pehuén; 2003.

3. Bateson G. Espíritu y naturaleza. Buenos Aires: Amorrortu Editores; 1990.

4. Guattari F, Deleuze G. Mil mesetas. Capitalismo y esquizofrenia. Valencia: Pre-textos; 1997.

5. Guattari F, Rolnik S. Micropolítica: Cartografías del deseo. Buenos Aires: Tinta Limón; 1996.

6. Foucault M. La "gouvernementalité". En: Defert D, Ewald F, editors. Dits et écrits 1954-1988. vol. II. Paris: Gallimard; 2001. p. 635-656.
7. Foucault M. Seguridad, territorio, población. Buenos Aires: Fondo de Cultura Económica; 2007.

8. Foucault M. Nacimiento de la biopolítica. Buenos Aires: Fondo de Cultura Económica; 2007.

9. Le Brass H. Naissance de la mortalité. Paris: GaIlimard, Seuil; 2000.

10. Petty W. Political Artihmetick [Internet]. McMaster University [citado 12 ene 2011] Disponible en: http://socserv2.socsci.mcmaster.ca/ econ/ ugcm/3II3/petty/poliarith.html.

11. Callon M. Why virtualism paves the way to political impotence: A reply to Daniel Miller's critique of The Laws of the Markets. Economic Sociology. 2006;6(2):3-20. 
12. Schumpeter J. Historia del análisis económico. México DF: Fondo de Cultura Económica; 1971.

13. Murillo A. La mortalidad urbana en Chile. Santiago de Chile: Imprenta y Encuadernación Roma; 1896.

14. Orrego-Luco A. La cuestión social en Chile. Anales de la Universidad de Chile. 1961(121122):43-55.

15. Commentz A. Estadísticas de mortalidad, natalidad y morbilidad en diversos países europeos y en Chile. En: Primer Congreso Nacional de Protección de la Infancia. Santiago de Chile; 1912. p. 313-334.

16. Covarrubias A. La estadística como base para combatir la mortalidad de los niños. En: Primer Congreso Nacional de Protección de la Infancia. Santiago de Chile; 1912. p. 345-352.

17. Behm H. Mortalidad infantil y nivel de vida. Santiago de Chile: Ediciones de la Universidad de Chile; 1962.

18. Muniesa F, Millo Y, Callon M. An introduction to market devices. Sociological Review. 2007;55(Supl 2):S1-S12.

19. Yates F. Giordiano Bruno y la tradición Hermética. Barcelona: Ariel Filosofía; 2005.

20. Foucault M. Les mots et les choses: Une archéologie de sciences humaines. Paris: Gallimard; 1966.

21. Eisenstein E. La imprenta como agente de cambio. México DF: Fondo de Cultura Económica; 2010.

22. Goody J. Cultura escrita en sociedades tradicionales. Buenos Aires: Gedisa; 2003.
23. Acuña C. El mundo subterráneo y la última carta de Nicolás Mascardi a su maestro Athanasius Kircher. En: Acuña C, editor. La curiosidad infinita de Athanasius Kircher. Santiago de Chile: Ocholibros; 2012

24. Goody J. El robo de la historia. Madrid: Akal; 2011.

25. Latour B. Reensamblar lo social: Una introducción a la teoría del actor red. Buenos Aires: Manantial; 2008.

26. Senra N. O saber e o poder das estatísticas. Rio de Janeiro: IBGE; 2005.

27. Desrosiéres A. Pour une sociologie historique de la quantification: L'Argument statistique I. Paris: Presses de l'Ecole des Mines; 2008.

28. Schwember $\mathrm{H}$. Las expulsiones de los jesuitas: O los fracasos del éxito. Santiago de Chile: J. C. Sáez Editor; 2005.

29. Latour B, Jensen $P$, Venturini $T$, Grauwin $S$, Boullier D. The whole is always smaller than its parts: a digital test of Gabriel Tardes' monads. British Journal of Sociology. 2012;63(4):590-615.

30. Theraulaz G, Bonabeau E. A brief history of stigmergy. Artificial Life. 1999;5(2):97-116.

31. Dewey J. El público y sus problemas. Madrid: Ediciones Mortata; 2004.

32. Peirce C. El hombre, un signo. Barcelona: Crítica; 1988

33. Whitehead A. Modos de pensamiento. Buenos Aires: Losada; 1944.

\section{FORMA DE CITAR}

Carvajal Y, Yuing T. Las estadísticas de salud no nacen de un repollo: jesuitas, aritméticas políticas, estigmergias y oligópticos. Salud Colectiva. 2013;9(1):91-102.

Recibido el 22 de agosto de 2012

Versión final presentada el 12 de noviembre de 2012

Aprobado 20 de diciembre de 2012 